\title{
STUDY OF SIGNIFICANCE OF PHASE MASK IMAGE IN ACUTE STROKE PATIENTS
}

\author{
Nerella Krishna Teja ${ }^{1}$, Dileep Reddy Ayapaneni ${ }^{1}$, Surekha Srikonda ${ }^{2}$ \\ Correspondence: krishna.teja666@gmail.com \\ ${ }^{I}$ Department of Radio-Diagnosis, Dr. D. Y. Patil Medical College, Hospital \& Research Centre, Pune, Maharashtra, India \\ ${ }^{2}$ Department of Radio-Diagnosis, Maharaja Institute of Medical Sciences (MIMS), Nellimarla, Vizianagaram District, Andhra Pradesh, \\ India
}

Article History:

Received: June 11, 2021

Accepted: October 4, 2021

Published: January 1, 2022

\section{Cite this as:}

Teja NK, Ayapaneni DR, Srikonda S. Study of significance of phase mask image in acute stroke patients. Malang Neurology Journal; 2022.8:6-10. DOI:

http://dx.doi.org/10.21776/ub.mnj .2022.008.01.2

\section{ABSTRACT}

Background: Phase images contains information regarding local susceptibility changes between the tissues, which can help measure the iron and other content which changes the local field. Typically, this information is ignored before looking at console. Susceptibility weighted imaging (SWI) is a magnetic resonance (MR) technique detects an early hemorrhagic transformation within the infarct to provide insight into cerebral hemodynamics following the stroke.

Objective: Significance of "phase mask imaging in differentiation of hemorrhage and calcifications" in acute stroke patients.

Methods: An observational non-interventional study carried out on 100 patients with stroke and headache symptoms. MRI Brain Stroke Profile with FLAIR, DWI, ADC, SWAN, and Phase mask sequences, done on 3T GE MRI scanner.

Results: All patients underwent MRI study with SWI sequence. Of 183 cases, 33\%(n=60) patients had microbleeds, 5\%(n=10) patients had granulomas, 32\%(n=58) patients had arterial thrombus with infarct, $11 \%(\mathrm{n}=20)$ patients had falx calcifications, $11 \%(\mathrm{n}=20)$ patients had intraparenchymal haemorrhage, and $8 \%(\mathrm{n}=15)$ patients had infarcts with haemorrhagic transformation. The sensitivity of phase imaging in the detection of calcification was $90 \%$.

Conclusion: Phase mask imaging plays an important role to detect intracranial calcifications and chronic microbleeds. Phase mask imaging acts as a supplement tool in acute stroke patients, which guides further management.

Keywords: Intra-cranial lesions, hemorrhage, infarct, susceptibility weighted imaging, artifacts.

\section{Introduction}

The presence of calcifications and hemorrhages characterizes many pathologies of brain parenchyma. It is important to differentiate hemorrhage and calcification for accurate diagnosis. SWI enhances the susceptibility induced contrast by combining the phase and magnitude. Recent advances allowed this method to be refined to utilize in various clinical applications. ${ }^{1}$ SWI can exploits the loss of signal intensity by susceptible substances due to inhomogeneity in the intrinsic magnetic field. The utility of SWI in the detection and characterization of various central nervous system lesions such as Traumatic Brain Injury, Cerebral Microbleeds, tumors, Vascular Malformations, Intracranial Calcifications, Infarcts, and Extra-axial Hemorrhage. ${ }^{2}$ In SWI, a phase mask from gradient recalled echo (GRE) MRI phase images are multiplied with the magnitude image., ${ }^{3,45}$ SWI has been used in cortical parcellation and to study the brain iron levels, calcification, and blood oxygenation of the cerebral venous system. ${ }^{6} \mathrm{SWI}$ is sensitive to deoxygenated blood and intracranial minerals and, hence it is used in diagnosing the intracranial hemorrhage, stroke, traumatic brain injury, neoplasm, and multiple sclerosis. ${ }^{1,7,8,5,9}$
Even though the phase and magnitude images were separate, they were critical information to avail the information available to radiologist.

\section{Methods}

\section{Source of data}

A non-interventional observational study includes the data from 100 patients who came to Department of Radiology for diagnosis referred from Emergency and Neurology departments. This observational study collected the data from the medical records of the patient's those usually visit to hospital for their disease management. The study obtained data retrospectively from the medical records, and hence ethical approval for this study was not required or waived. ${ }^{10}$ All patients undergoing brain imaging at our centre from all ages with symptoms of stroke and headache were analysed. Data from patients with claustrophobic, unwilling, with metal/cochlear implants/pacemakers, and pregnant women were excluded.

\section{MRI protocol}

All patients were subjected to brain MRI examination using GE Signa 3 Tesla MRI scanner. MRI Brain Stroke Profile 
with FLAIR, DWI, ADC, SWAN, and Phase mask sequences, done.

\section{Image reconstruction in $\mathrm{SW}$ imaging}

HP filter was applied to delete the low spatial frequency components of background by using 64 X 64 lowpass filter divided into the original phase image (matrix size $512 \mathrm{X}$ 512). The door was open to differentiate one type of tissue to another type, based on it's susceptibility.

SWI sequence generates raw phase image, and magnitude image. The raw phase image contains background unwanted low frequency information. The filtered phase image is converted into a phase mask and combined with the magnitude image to form final SWI. SWI sequence generated phase, magnitude, SWI axial, and thick minimum intensity projection (mIP) images.

\section{Image and data analysis}

Two independent radiologists performed the qualitative evaluation of focal calcification and chronic microbleeds to assess signal intensities independently. Finally compared their findings to analyse the role of SWI in better lesion detection and characterization.

\section{Results}

All patients underwent MRI study with SWI sequence. Of 183 cases, 33\% $(\mathrm{n}=60)$ patients had microbleeds, $5 \%(\mathrm{n}=10)$ patients had granulomas, $32 \%(\mathrm{n}=58)$ patients had arterial thrombus with infarct, $11 \%(\mathrm{n}=20)$ patients had falx calcifications, $11 \%(\mathrm{n}=20)$ patients had intraparenchymal haemorrhage, and $8 \%(n=15)$ patients had infarcts with haemorrhagic transformation

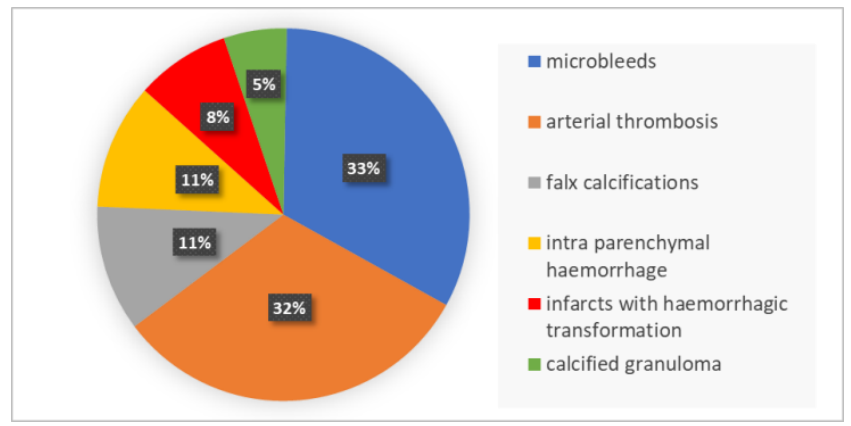

Figure 1. Phase Mask MR Image Analysis of Various Acute Stroke Patients.

\section{Cerebral Microbleeds}

We encountered 60 patients where SWI revealed multiple foci of 'blooming' suggestive of microbleeds, out of whom 46 patients had a positive history of hypertension. The radiological diagnosis in these patients was chronic hypertensive encephalopathy/Amyloid angiopathy with multiple microbleeds (Figure 2). T2- weighted images reveal generalized prominence of the sulci, fissures, and cisterns with chronic lacunar infarcts in bilateral basal ganglia (solid black arrow) and non-specific white matter changes in the periventricular region and corona radiate (highlighted). T1-weighted images corroborate findings on T2-weighted images. SWI reveals foci of magnetic susceptibility in bilateral basal ganglia, thus confirming chronic hypertensive encephalopathy (Figure 3).

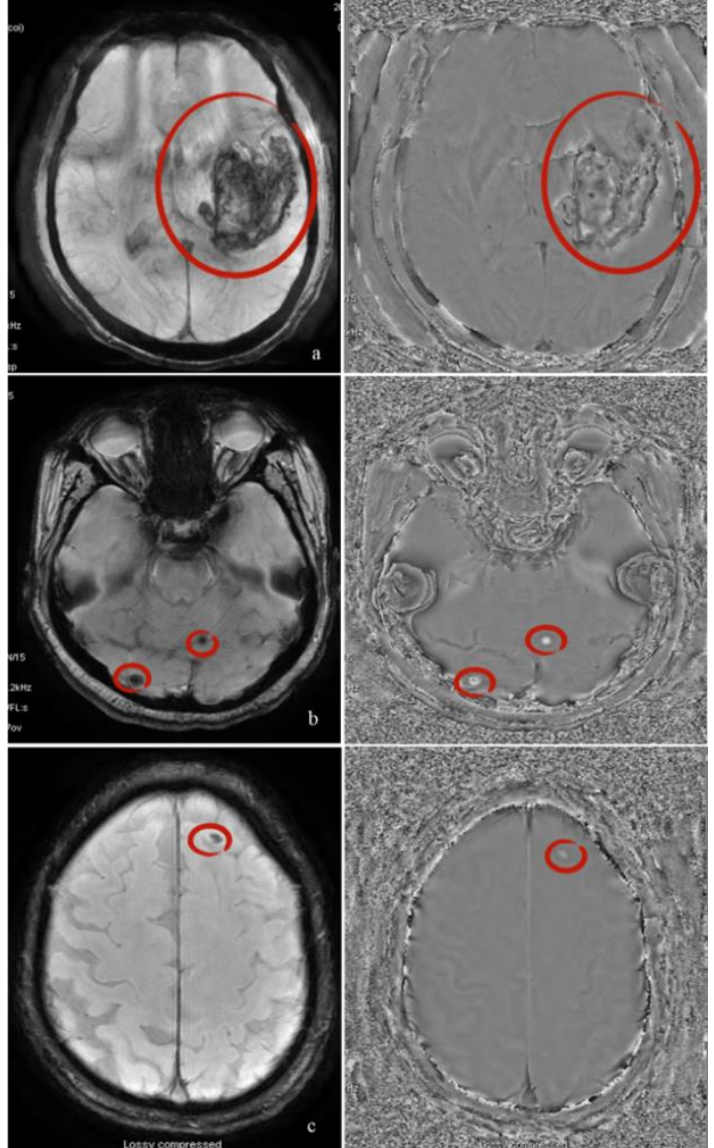

Figure 2. Magnitude and phase image information of acute stroke patients. (a). Case 1-Intraparenchymal haemorrhage in a patient with right hemiparesis. (b). Case 3-calcified granuloma in a patient with left hemiparesis-Mimic of cerebral microbleed. (c). Case 4-calcified granuloma in a hypertensive patient-mimic of microbleed.

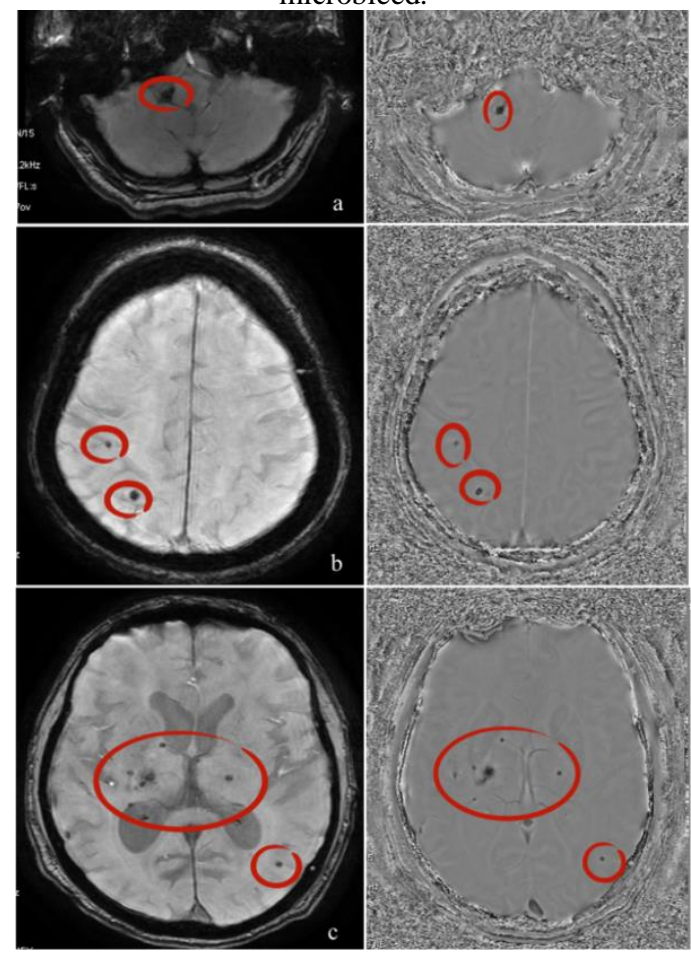

Figure 3. Magnitude and phase image information of acute stroke patients. (a). Case 8-Right vertebral artery(V4) thrombus. (b). Case 9-microbleeds in a patient with cerebellar infarct-Mimic of calcified granulomas. (c) Case 10 microbleeds in a patient with hypertension and diabetes-Mimic of calcified granulomas 


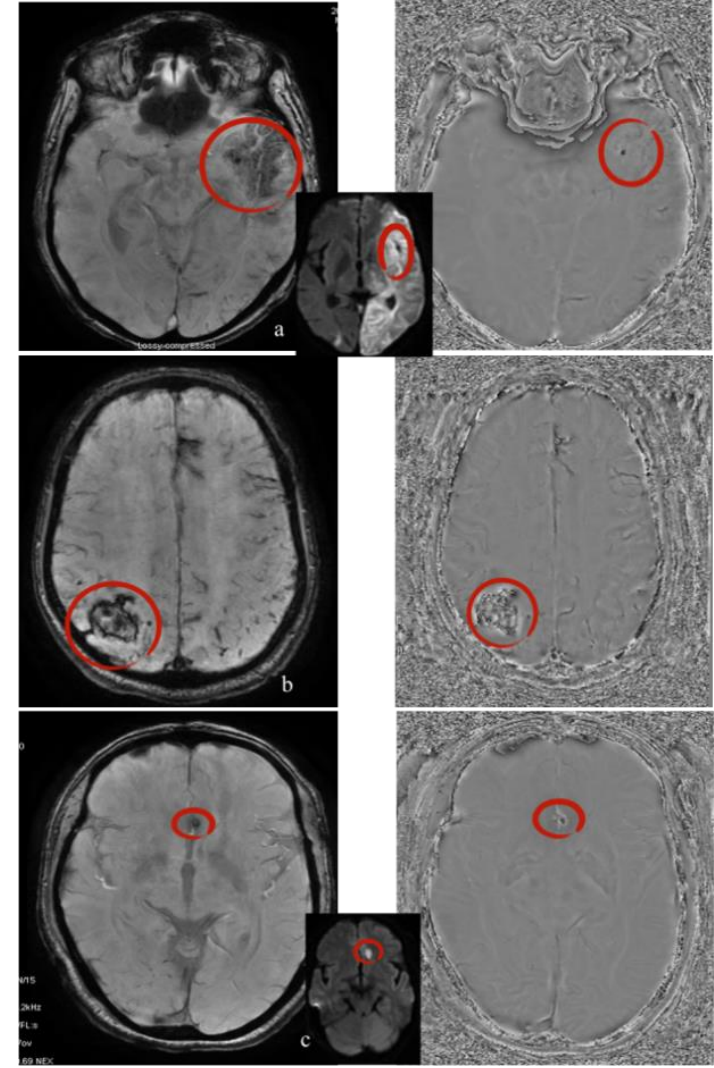

Figure 4. Magnitude and phase image information of acute stroke patients. (a). Case 5-Arterial infarct with haemorrhagic transformation. (b). Case 6-Venous infarct with haemorrhagic transformation showing thrombosed cortical vein. (c). Case 7Left ACA thrombus.
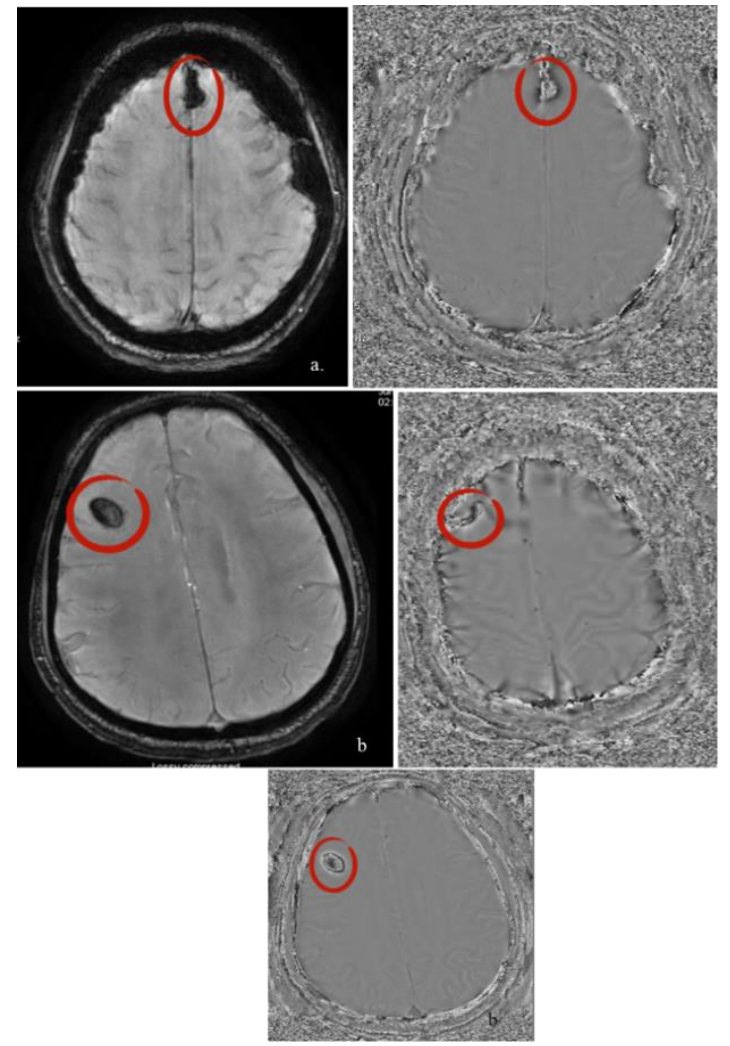

Figure 5. Magnitude and phase image information of acute stroke patients. (a). Case 11 Falx calcification in a patient with suspected venous thrombosis-Mimic of superior saggital sinus thrombus .(b).

Case 12-Venous infarct with haemorrhagic transformation showing thrombosed cortical vein. Case 12-Venous infarct with haemorrhagic transformation showing thrombosed cortical vein

\section{Infarcts}

Among the patients with acute and subacute infarct findings on conventional sequences, $32 \%(\mathrm{n}=58)$ patients had arterial thrombus with infarct detected by SWI. Among these infarcts, the hemorrhagic transformation was detected in 15 patients on SWI as well as conventional sequences. Both conventional sequences and SWI were equally sensitive in detecting hematomas (Figure 4).

\section{Calcifications}

20 patients were diagnosed with calcified granulomata and out of these SWI was useful in detecting 18 lesions that were indiscernible on other sequences. In remaining 3 patients, low signal intensity was perceived on the conventional sequences (Figure 5).

\section{Discussion}

Our study results show that mask imaging can give critical information regarding differentiation of hemorrhage and calcification that could be significant in assessing acute stroke.

Haacke et al. ${ }^{5}$ described that the phase images provide the accurate contrast between the gray and white matter and materials with susceptibilities which is different from the nearby tissue, such as hemorrhage, calcification, and blood vessels carrying deoxygenated blood.

Chronic microbleeds are asymptomatic with diameter $<5$ $10 \mathrm{~mm}$ small bleeds. SWI proved as high sensitive to diagnose the chronic microbleeds. In our study, we identified 60 patients, where SWI revealed the multiple foci of 'blooming' suggestive of microbleeds, out of them 46 patients had a positive history of hypertension. Whereas, a study by Sahdev R et al. ${ }^{11}$ encountered 32 patients where SWI revealed multiple foci of 'blooming' suggestive of cerebral microbleeds.

Regarding the traumatic brain injury (TBI), the detection of huge intracranial hemorrhage has always been important for management. $^{12}$ The paramagnetic properties of deoxygenated hemoglobin and blood degradation products (like intra-cellular hemosiderin and methemoglobin) make easy to exploit the magnetic susceptibility effects. Tong et al. ${ }^{13}$ found SWI to detect six times the number of hemorrhagic diffuse axonal injury lesions compared to T2*weighted 2D GRE imaging in seven children with traumatic brain injury. Thus, while larger hemorrhages may be easily visualized on $\mathrm{CT}^{14}$ and conventional MRI sequences, SWI is the only sequence available to detect smaller hemorrhagic lesions caused by shearing forces. Also, SWI has proved a useful adjunct in confirming the presence of extra-axial hemorrhage. ${ }^{15}$

Regarding Vascular Malformations, SWI is superior to the conventional sequences in visualizing low flow vascular malformations like telangiectasias and venous anomalies. ${ }^{16}$ These malformations are usually not visible even on conventional angiography. ${ }^{17,18}$

In our study, among the patients with acute and subacute infarct findings on conventional sequences, $32 \%$ of patients had arterial thrombus with infarct detected by SWI. Among these infarcts, the hemorrhagic transformation is observed in 15 patients on SWI as well as conventional sequences. Whereas study by Sahdev $\mathrm{R}$ et al. ${ }^{11}$ revealed that 277 
patients revealed positive findings on SWI. Among these, hemorrhagic transformation identified in 189 patients on SWI and conventional sequences.

Regarding Intracranial Calcifications, discrimination between the iron and calcium was difficult even on SWI sequences. In our study, 20 patients were diagnosed with calcified granulomata, and out of these, SWI helped detect 18 lesions that were indiscernible on other sequences. In the remaining two patients, low signal intensity was perceived on the conventional sequences. Whereas study by Sahdev R et al. ${ }^{11}$ revealed, 128 patients were diagnosed with calcified granulomata, and out of these, SWI was useful in detecting 46 lesions that were indiscernible on other sequences. Zhu WZ et al. ${ }^{19}$ described that calcification and hemorrhage manifested the low signal on SWI. They presented opposite signal features on the corrected phase images.

Detecting a hemorrhagic component in a region of the infarct is a contraindication for IV thrombolysis ${ }^{20}$ demonstration of an arterial thrombo-embolic clot in acute stroke to differentiate it from atheromatous calcification. Phase mask image accurately detects micro-hemorrhages from calcified granulomas, in predicting the probability of potential hemorrhagic transformation. Falx calcification is a significant mimic on SWI images, differentiated from venous sinus thrombosis, where phase mask image plays a major role. Acute stroke patients with intra-parenchymal bleeding must be differentiated from calcifications. Thrombosed cortical veins are better appreciated in venous infarcts which helps in assessing the prognosis.

\section{Conclusion}

Phase mask imaging plays a major role in the differentiation of calcifications from the haemorrhages. Phase mask imaging acts as a supplement tool in acute stroke patient which guides further management.

\section{Conflict of Interest}

No conflict of interest declared by authors.

\section{Acknowledgement}

The researchers would like to acknowledge 1Department of Radio-Diagnosis, Dr. D. Y. Patil Medical College, Hospital \& Research Centre and Department of Radio-Diagnosis, MIMS for supporting this research.

\section{References}

1. Halefoglu AM, Yousem DM. Susceptibility weighted imaging: Clinical applications and future directions. World journal of radiology; 2018 Apr 28;10(4):30. DOI: $10.4329 /$ wjr.v10.i4.30

2. Gratz PP, El-Koussy M, Hsieh K, von Arx S, Mono ML, Heldner MR, Fischer U, Mattle HP, Zubler C, Schroth G, Gralla J. Preexisting cerebral microbleeds on susceptibility-weighted magnetic resonance imaging and post-thrombolysis bleeding risk in 392 patients. Stroke; 2014 Jun;45(6):1684-8. DOI: 10.1161/STROKEAHA.114.004796

3. Barnes SR, Haacke EM. Susceptibility-weighted imaging: clinical angiographic applications. Magnetic resonance imaging clinics of North America. 2009 Feb 1;17(1):47-61. DOI: 10.1016/j.mric.2008.12.002

4. Deistung A, Barnes S, Ge Y, Reichenbach JR. Susceptibility weighted imaging at ultrahigh magnetic fields. Susceptibility weighted imaging in MRI. Basic Concepts and Clinical Applications.; 2011 Jan 24:32949.DOI: https://doi.org/10.1002/9780470905203.ch20

5. Haacke EM, Ye Y. The role of susceptibility weighted imaging in functional MRI. Neuroimage; 2012. 62:923-929. DOI: 10.1016/j.neuroimage.2012.01.020.

6. Robinson R.J, Bhuta S. Susceptibility-weighted imaging of the brain: Current utility and potential applications. Journal of Neuroimaging: Official Journal of the American Society of Neuroimaging. 2011. 21:e189-e204. DOI: 10.1111/j.15526569.2010.00516.x

7. Azad R, Mittal P, Malhotra A, Gangrade S. Detection and differentiation of focal intracranial calcifications and chronic microbleeds using MRI. Journal of Clinical and Diagnostic Research; 2017 May;11(5):TC19.

DOI: $10.7860 / \mathrm{JCDR} / 2017 / 24076.9846$

8. Haacke EM, Reichenbach JR, Editors. Susceptibility weighted imaging in MRI: Basic concepts and clinical applications. John Wiley \& Sons; 2014 Mar 25.

DOI: $10.1002 / 9780470905203$

9. Mittal S, Wu Z, Neelavalli J, Haacke E. Susceptibility-weighted imaging: Technical aspects and clinical applications, part 2. American Journal of Neuroradiology; 2009;30:232-252. DOI: 10.3174/ajnr.A1461

10. Claudot F, Alla F, Fresson J, Calvez T, Coudane H, Bonaïti-Pellié C. Ethics and observational studies in medical research: Various rules in a common framework. International journal of epidemiology. 2009 Aug 1;38(4):1104-8. DOI: 10.1093/ije/dyp164

11. Sahdev R, Rao A, Yadu N, Shukla Y. Role of susceptibility weighted imaging in characterization of intra-cranial lesions. Int J Med Res Rev; 2016. 4(11):2006-2014.

DOI: $10.17511 /$ ijmrr.2016.i11.19

12. Tsivgoulis G, Zand R, Katsanos AH, et al. Risk of symptomatic intracerebral hemorrhage after intravenous thrombolysis in patients with acute ischemic stroke and high cerebral microbleed burden: A meta-analysis. JAMA neurology; 2016. Jun 1;73(6):675-83. DOI: 10.1001/jamaneurol.2016.0292

13. Tong KA, Ashwal S, Holshouser BA, et al. Diffuse axonal injury in children: Clinical correlation with hemorrhagic lesions. Ann Neurol; 2004. 56:36-50. DOI: 10.1002/ana.20123

14. Kızılgöz V, Avcı S, Sivrioğlu AK, et al. Evaluation of the canadian ct head rule for analyzing diagnostic accuracy in patients with minor head trauma. Malang Neurology Journal; 2019 Apr 29;5(2):68-75.

DOI: 10.21776/ub.mnj.2019.005.02.4

Sehgal V, Delproposto Z, Haddar D, et al. Susceptibility-weighted imaging to visualize blood products and improve tumor contrast in the study of brain masses. J Magn Reson Imaging ; 2006. 24:4151. DOI: $10.1002 /$ jmri.20598 
15. New PFJ, Ojeman RG, Davis KR, et al. MR and CT of occult vascular malformations of the brain. Am $\mathrm{J}$ Neuroradiol; 1986. 7:771-779. DOI: 10.2214/ajr.147.5.985

16. Marchal G, Bosmans H, Fraeyenhoven LV, Wilms G, Hecke PV, Plets C.A.L.B. Intracranial vascular lesions. Optimization and clinical evaluation of threedimensional time-of-flight MR angiography. Radiology; 1990. 175:443-448.

DOI: 10.1148/radiology.175.2.2326471

17. Santhosh K, Kesavadas C, Thomas B, Gupta AK, Thamburaj K, Kapilamoorthy T. Susceptibility weighted imaging: A new tool in magnetic resonance imaging of stroke. Clin Radiol; 2009. 64(1):74-83. DOI: 10.1016/j.crad.2008.04.022

18. Zhu WZ, Qi JP, Zhan CJ, Shu HG, Zhang L, Wang $\mathrm{CY}$, et al. Magnetic resonance susceptibility weighted imaging in detecting intracranial calcification and hemorrhage. Chin Med J; 2008. 121:2021-25.

19. Rachmawati D, Ningsih DK, Andarini S. Factors affecting the knowledge about stroke risks and early symptoms in emergency department East JavaIndonesia. Malang Neurology Journal; 2020. Jan 30;6(1):11-9.

DOI: 10.21776/ub.mnj.2020.006.01.3 